\title{
Intracameral bevacizumab as an adjunct to trabeculectomy: a 1-year prospective, randomised study
}

\author{
Evelien Vandewalle, ${ }^{1}$ Luís Abegão Pinto, ${ }^{2,3}$ Tine Van Bergen, ${ }_{1}{ }^{4}$ Leigh Spielberg, ${ }^{5}$ \\ Steffen Fieuws, ${ }^{6}$ Lieve Moons, ${ }^{7}$ Werner Spileers, ${ }^{1}$ Thierry Zeyen, ${ }^{1}$ \\ Ingeborg Stalmans ${ }^{1,4}$
}

1 Department of Ophthalmology, University Hospitals Leuven Leuven, Belgium

${ }^{2}$ Department of Pharmacology and Neurosciences of Faculty

of Medicine of Lisbon

University, Lisbon, Portugal

${ }^{3}$ Department of Ophthalmology,

Centro Hospitalar Lisboa

Central, Lisbon, Portugal

${ }^{4}$ Laboratory of Ophthalmology, Katholieke Universiteit Leuven, Leuven, Belgium

5 Department of Ophthalmology, Rotterdam Eye Hospital, Rotterdam, The Netherlands

${ }^{6}$-Biostat, Katholieke Universiteit Leuven, Belgium Department of Animal physiology and Neurobiology, Katholieke Universiteit Leuven, Leuven, Belgium

\section{Correspondence to} Professor I Stalmans Department of Ophthalmology, University Hospitals Leuven, Campus St Raphaël, Kapucijnenvoer 33, Leuven B-3000, Belgium; ingeborg.stalmans@uzleuven.be

EV, LAP contributed equally to the study and therefore should be considered both as first author

Received 4 July 2013 Revised 24 August 2013

Accepted 14 September 2013 Published Online First 24 October 2013

\footnotetext{
To cite: Vandewalle $\mathrm{E}$, Abegão Pinto L, Van Bergen $\mathrm{T}$, et al. $\mathrm{Br}$ J Ophthalmol 2014;98: 73-78.
}

\section{ABSTRACT}

Aims To investigate the efficacy and safety of a single intracameral bevacizumab injection to improve the outcome of trabeculectomy.

Methods A 12-month, prospective, randomised double-masked, placebo-controlled trial. Patients with medically uncontrolled open-angle glaucoma scheduled for a primary trabeculectomy were recruited and randomised to receive $50 \mu \mathrm{L}$ of either bevacizumab (1.25 mg) or placebo (balanced salt solution) peroperatively. Absolute success was defined as intraocular pressure (IOP) $\leq 18 \mathrm{~mm} \mathrm{Hg}$ and $>5 \mathrm{~mm} \mathrm{Hg}$ with at least $30 \%$ reduction from baseline and no loss of light perception. Success through the use of additional medical and/or surgical IOP-lowering treatments was defined as qualified success.

Results 138 patients completed a 12-month follow-up 69 of whom were in the bevacizumab treated group. IOP at 1 year postoperatively was significantly lower than baseline (placebo: $25.6 \pm 9.9 \mathrm{~mm} \mathrm{Hg}$ vs 11.5 $\pm 3.9 \mathrm{~mm} \mathrm{Hg}, p<0.01$; bevacizumab: $24.8 \pm 8.1 \mathrm{~mm} \mathrm{Hg}$ vs $11.9 \pm 3.8 \mathrm{~mm} \mathrm{Hg}, p<0.01$ ), with no difference between treatment groups $(p=0.69)$. However, absolute success was higher in the bevacizumab group $(71 \%$ vs $51 \%, p=0.02$ ), with the need for IOP-lowering interventions (needlings) being lower in this group (12\% vs $33 \%, p=0.003)$. Complication rates were low and comparable between groups.

Conclusions Peroperative administration of intracameral bevacizumab significantly reduces the need for additional interventions during the follow-up of patients undergoing trabeculectomy.

\section{INTRODUCTION}

The failure of glaucoma filtration surgery due to excessive wound healing remains a challenge. Numerous antifibrotic agents have been considered, ${ }^{1}{ }^{2}$ but none has replaced mitomycin $\mathrm{C}$ (MMC) and 5-fluorouracil (5-FU) in clinical practice, despite the ongoing debate of their risk of complications. ${ }^{3} 4$ Therefore, there is still a need for adjunctive therapeutic strategies to improve surgical outcome, to prevent filtration failure, and to find a good balance between safety and efficacy.

Vascular endothelial growth factor (VEGF) is known to play a role in the formation of pathologic angiogenesis in tumour growth, inflammation, wound healing, and various ocular diseases. ${ }^{5} 6$ The ability of anti-VEGF agents (such as bevacizumab at a standard $1.25 \mathrm{mg} / 0.05 \mathrm{~mL}$ concentration) to inhibit the proliferation and migration of Tenon fibroblasts has been well documented. ${ }^{7}{ }^{8}$ The use of anti-VEGF agents may be particularly interesting in glaucoma patients, since they have increased concentrations of VEGF in the aqueous humour. ${ }^{79}$

Several animal studies and human case series have been published with the use of anti-VEGF agents in filtering surgery and have consistently demonstrated their favourable effects on surgical outcomes. ${ }^{10-12}$ While these studies have shown the safety of the use anti-VEGF agents in intraocular surgery, evidence from large prospective randomised clinical trials to demonstrate their efficacy for improving surgical outcomes of trabeculectomy is still lacking.

This study sought to further explore this promising modality with a prospective, randomised, doublemasked, placebo-controlled approach to compare the effect of a single, adjunctive intracameral administration of bevacizumab $(1.25 \mathrm{mg} / 0.05 \mathrm{~mL})$ with placebo (balanced salt solution (BSS)) on the surgical outcome of primary trabeculectomy in patients with open-angle glaucoma.

\section{PATIENTS AND METHODS \\ Design}

This prospective, randomised, double-masked, placebo-controlled experimental trial was approved by the institutional review board of the University Hospitals Leuven and adhered to the tenets of the Declaration of Helsinki. All eligible patients who agreed to participate in the study signed an informed consent before enrolment. This study was registered on eudract.emea.europe.eu (registration number 2009-009038-33).

\section{Patients}

Between April 2009 and November 2010, consecutive glaucoma patients scheduled for primary trabeculectomy were recruited at the Department of Ophthalmology of the University Hospitals Leuven, Belgium. Glaucoma was defined as having characteristic optic disc damage and visual field defects. Indications for surgery were based on: (1) intraocular pressure (IOP) associated with high probability of glaucoma progression; (2) glaucomatous visual field loss or changes of the optic disc indicative of progressive glaucoma damage; or (3) allergy or intolerance to current maximal topical therapy.

Exclusion criteria were age $<18$ years, history of ocular trauma or eye disease (except glaucoma) that 
could not be accounted for by refractive error, best refracted visual acuity less than -1.18 on the ETDRS (Early Treatment Diabetic Retinopathy Study) scale, history of previous intraocular surgical interventions other than uneventful clear-corneal cataract surgery, severe cardiovascular disease including a history of acute myocardial infarction or stroke, untreated brain cancer, inflammatory bowel disease, recent or planned surgery (other than the trabeculectomy), unhealed wounds, pregnancy, childbearing potential without the use of adequate contraception, and a known allergy to bevacizumab.

Randomisation was performed by the pharmacist using a computer-based randomisation programme (http://www. randomization.com—accessed 15 March 2009). The pharmacist provided the investigators with masked syringes of bevacizumab or placebo, as well as a sealed randomisation list for use in case of emergency. As the two solutions are visually indistinguishable, the observers remained masked during surgery and during the entire study period. Other than for safety reasons, the unmasking of the subjects would only take place after the 12-month period was completed.

\section{Observation procedure}

The following information was recorded: age, gender, baseline IOP (mean of last two recordings on separate days and hours), past ocular history, preoperative visual acuity, visual field mean deviation, central corneal thickness, and duration of disease. IOP was measured by Goldmann applanation tonometry. Two measurements were taken by masked observers and averaged to determine the mean IOP if two values were within $2 \mathrm{~mm} \mathrm{Hg}$. A third measurement was taken if the difference between the first two determinations was $>2 \mathrm{~mm} \mathrm{Hg} .{ }^{13}$

The number of ocular hypotensive medications was also recorded. Fixed combinations were documented according to the number of active ingredients.

\section{Surgical technique}

Surgeries were performed under general or retrobulbar anaesthesia by two experienced surgeons (IS, TZ) using a modified Moorfields technique. ${ }^{14}$ A silk 8-0 corneal traction suture was placed, and a fornix-based conjunctival flap was dissected. A half-thickness scleral flap measuring $5 \times 4 \mathrm{~mm}$ was delineated with a $30^{\circ}$ blade (Micro Feather, Feather safety razor Co., Osaka, Japan) and dissected with a crescent knife (Alcon). MMC was used when the target pressure (based on a $30 \%$ reduction from baseline) was calculated to be $<14 \mathrm{~mm} \mathrm{Hg}$. In each of those cases, MMC $(0.2 \mathrm{mg} / \mathrm{mL})$-soaked sponges were applied for 2 min under both the conjunctival and the scleral flaps and then abundantly rinsed. Two diagonal flap sutures (nylon 10-0) were pre-installed. A corneal paracentesis was made using a $30^{\circ}$ blade and a ocular viscoelastic device (Viscoat; Alcon, Puurs, Belgium) was injected into the anterior chamber. A trabeculectomy was performed using the Khaw titanium punch of $0.5 \mathrm{~mm}$ (No. 7-102: Duckworth and Kent, Hertfordshire, UK), followed by a peripheral iridectomy. The two fixed flap sutures were tight and the conjunctiva was closed with two nylon 10-0 sutures at the limbus using a 'purse string' technique. Finally, after rinsing out the viscoelastic substance, the anterior chamber was sealed by hydration of the side port and the study medication $(50 \mu \mathrm{L}$ bevacizumab at a concentration of $25 \mathrm{mg} / \mathrm{mL}$ ) or placebo (BSS) was injected intracamerally through the paracentesis using a single-use 30 -gauge needle. At the end of the surgery, a subconjunctival injection of betamethasone (Celeston chronodose, Schering-Plough NV/SA, Brussels, Belgium) was administered. A topical preparation containing tobramycin and dexamethasone (Tobradex; Alcon) was then applied as an ointment and was continued as drops four times daily for 8 weeks and then tapered over 3 weeks. In case of allergy to benzalkonium chloride, preservative-free dexamethasone (Dexamethasone monofree, Théa Pharma, Wetteren, Belgium) and ofloxacin (Trafloxal edo, Dr Mann Pharma, Berlin, Germany) were prescribed.

\section{Follow-up evaluations}

Patients were examined on day 1 ; at weeks 1,2 , and 4 ; and at months 3, 6, and 12 after trabeculectomy. All patients underwent a comprehensive ophthalmic examination that included measurements of best-corrected visual acuity, slit-lamp examination including a Seidel test, IOP measurement, and fundus biomicroscopy with a 90-diopter lens. The number of postoperative IOP-lowering medications, intra- and postoperative complications, and surgical interventions were also recorded.

\section{Outcome measurements}

The primary outcome was absolute success, defined as at least $30 \%$ reduction in IOP from baseline, with an IOP $\leq 18 \mathrm{~mm} \mathrm{Hg}$ and $>5 \mathrm{~mm} \mathrm{Hg}$ and no loss of light perception. The secondary outcome was qualified success, which refers to achieving these criteria through the use of additional medical and/or surgical IOP-lowering treatments (except early postoperative bleb massage and suture lysis, which are office-based manipulations that can be used to titrate the IOP gradually). Specifically, the need for a new filtering procedure (trabeculectomy or aqueous humour drainage device implantation) was a criterion for surgical failure at any time point.

\section{Sample size}

The sample size calculations were based on a two-sided Pearson $\chi^{2}$ test for two proportions. Based on previous success rates published by our group using the same criteria, ${ }^{15}$ setting the $\alpha$ error to $5 \%$ and power at $80 \%$ would require the recruitment of 62 patients per group to identify a $25 \%$ difference in absolute success. Considering a possible dropout rate of up to 15\%, 144 patients were enrolled.

\section{Statistical analysis}

Comparisons between treatment groups were performed using the two-sided Student $t$ test for continuous variables and Fisher's exact test for categorical variables. Primary and secondary outcome variables were compared between the two groups using an intention to treat analysis. This comparison was performed based on an OR in a stratified $3 \times 2$ table. The two-sided $\mathrm{p}$ value from an exact test for the OR was calculated, and an exact two-sided 95\% CI was constructed. Treatment comparisons using both success definitions were assessed with the stratified Kaplan-Meier survival log-rank test. The analyses were performed using SAS software (V.9.2; SAS Inc, Cary, North Carolina, USA). A value of $\mathrm{p}<0.05$ was considered significant.

\section{RESULTS}

\section{Recruitment and retention}

Between April 2009 and November 2010, 144 patients were enrolled. Randomisation assigned 72 patients to the bevacizumab group and 72 patients to the control group. In the overall study, 138 patients reached the 12-month time point, and six patients $(4.2 \%)$ were lost to follow-up at 1 year (figure 1 ). 
Figure 1 Flowchart of patient progress in the 12-month study.

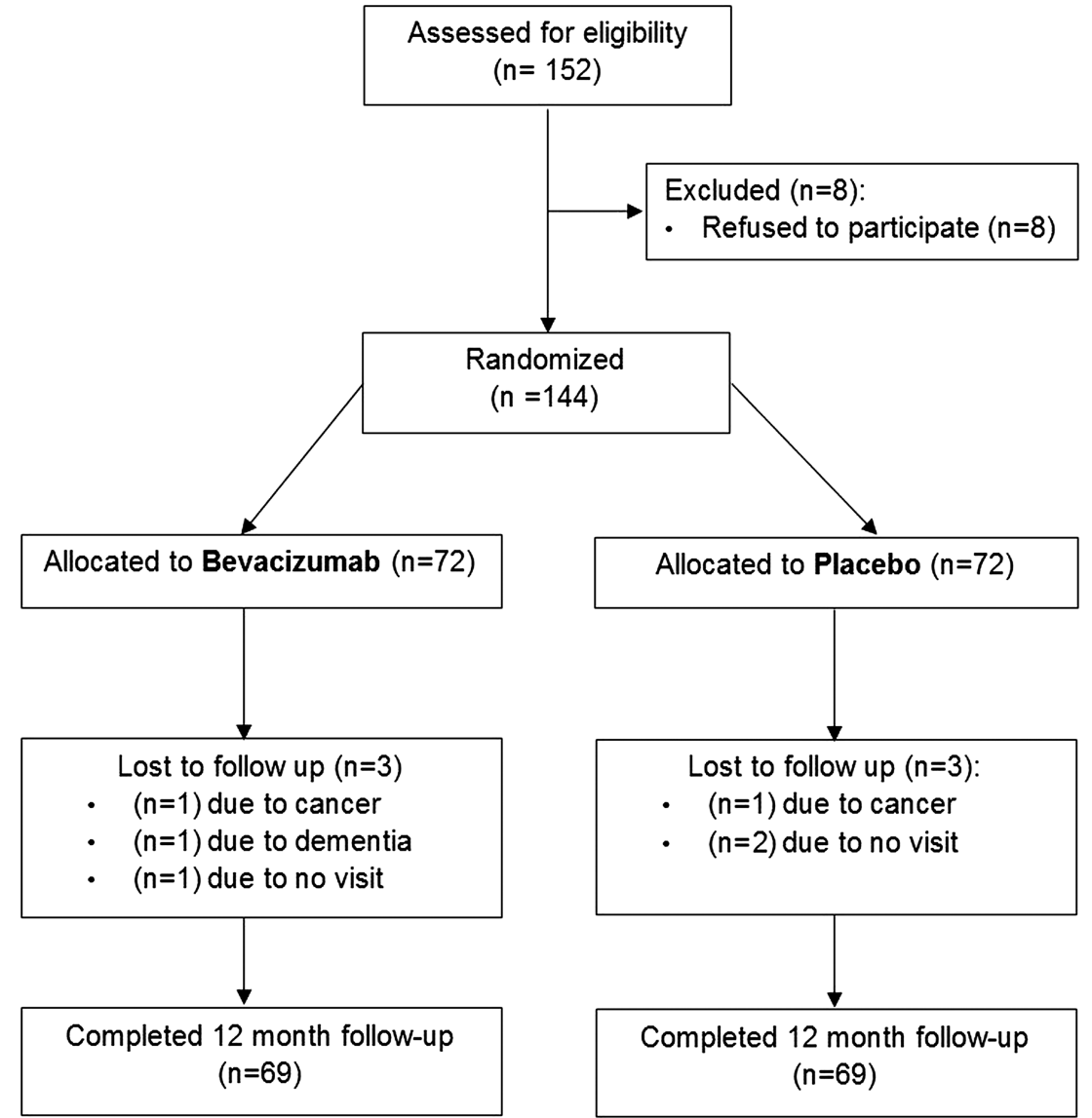

\section{Baseline characteristics}

The baseline characteristics of the study patients are summarised in table 1. The two groups (bevacizumab vs placebo-treated) were similar when initiating the study, including the proportion of patients in which MMC was used $(\mathrm{p}=0.87)$.

\section{IOP lowering}

IOP was effectively reduced in both groups at the 12-month visit when compared to baseline (placebo: $25.6 \pm 9.9$ vs $11.5 \pm 3.9$,

Table 1 Baseline characteristics of participating patients

\begin{tabular}{lll}
\hline & $\begin{array}{l}\text { Bevacizumab } \\
(\mathbf{n}=72)\end{array}$ & $\begin{array}{l}\text { Placebo } \\
(\mathbf{n}=72)\end{array}$ \\
\hline Age (years) & $69 \pm 10$ & $69 \pm 10$ \\
Female/male gender* & $40 / 32$ & $42 / 30$ \\
IOP (mm Hg) & $24.8 \pm 8.1$ & $25.6 \pm 9.9$ \\
Previous uneventful cataract surgery* & 14 & 17 \\
Visual acuity (logMar) & $0.2 \pm 0.2$ & $0.2 \pm 0.2$ \\
Visual field MD (dB) & $-11.5 \pm 8$ & $-10.2 \pm 7$ \\
CCT ( $\mu$ m) & $545 \pm 37$ & $552 \pm 37$ \\
No. of preoperative medications & $2.5 \pm 1.1$ & $2.4 \pm 1.2$ \\
Time between diagnosis and surgery & $7.2 \pm 6.5$ & $7.4 \pm 6.4$ \\
(years) & & $37 / 35$ \\
Allocation to MMC/no MMC subgroups & $35 / 37$ & \\
\hline
\end{tabular}

Comparison between the baseline characteristics of the two experimental groups. Student $t$ test was performed in all comparisons except when noted $\left({ }^{*}\right)$, in which Fisher's exact test was used. $p>0.05$ in all comparisons. Continuous variables depicted as mean $\pm S D$

CCT, central corneal thickness; IOP, intraocular pressure; logMar, logarithm of the minimum angle of resolution; MD, mean defect; $M M C$, mitomycin $C$. $\mathrm{p}<0.01$; bevacizumab: $24.8 \pm 8.1$ vs $11.9 \pm 3.8, \mathrm{p}<0.01)$. No difference in IOP was detected between bevacizumab and placebo-treated patients $(p=0.69)$. Table 2 depicts the IOP levels throughout the study visits in both groups, stratified by the use of an antimetabolite (ie, simple and MMC-augmented trabeculectomy).

\section{Success rates}

Absolute and qualified success rates according to the predetermined definition are presented in table 3. There was a significant difference in absolute success rate $(51 \%$ vs $71 \%$, $\mathrm{p}=0.02)$ but not in qualified success rate $(86 \%$ vs $87 \%$, $\mathrm{p}=0.99$ ) between placebo- and bevacizumab-treated eyes. Kaplan-Meier survival curve log-rank tests identified significant differences between the two groups when assessing absolute success $(p<0.01)$, but not when assessing qualified success $(p=0.84)$ (figure 2). A subgroup analysis on the use of bevacizumab in simple or MMC-augmented trabeculectomy was also performed. Despite no overall differences in the success rates at 12 months in any of the subanalyses (table 3), log-rank tests showed that bevacizumab improved the absolute success survival curves in both types of trabeculectomy (simple: $p=0.045$; MMC-augmented: $p=0.03$ ) but had no effect on the qualified success curves (simple: $p=0.60$; MMC-augmented: $\mathrm{p}=0.31$ ).

Failures $(n=19)$ were most frequently related to IOP above target $(n=17)$, with only two patients developing long-term hypotony. These patients were the only ones to sustain a vision loss $>10$ EDTRS letters, although no patients lost light perception. Causes for the loss of six patients during follow-up are described in figure 1 . 
Table 2 Intraocular pressure and IOP-lowering medication throughout the study visits

\begin{tabular}{|c|c|c|c|c|}
\hline & \multicolumn{2}{|l|}{ Bevacizumab } & \multicolumn{2}{|l|}{ Placebo } \\
\hline & $\begin{array}{l}\text { Augmented } \\
(\mathrm{n}=32)\end{array}$ & $\begin{array}{l}\text { Simple } \\
(n=37)\end{array}$ & $\begin{array}{l}\text { Augmented } \\
(\mathrm{n}=34)\end{array}$ & $\begin{array}{l}\text { Simple } \\
(n=35)\end{array}$ \\
\hline \multicolumn{5}{|l|}{ D1 } \\
\hline IOP & $9.0 \pm 3.9$ & $11.8 \pm 4.5$ & $8.2 \pm 5.2$ & $8.8 \pm 4.7$ \\
\hline Medication & $0.0 \pm 0.0$ & $0.0 \pm 0.0$ & $0.0 \pm 0.0$ & $0.0 \pm 0.0$ \\
\hline \multicolumn{5}{|l|}{ W1 } \\
\hline IOP & $8.2 \pm 3.7$ & $9.3 \pm 3.6$ & $7.3 \pm 3.8$ & $7.7 \pm 3.1$ \\
\hline Medication & $0.0 \pm 0.0$ & $0.0 \pm 0.0$ & $0.0 \pm 0.0$ & $0.0 \pm 0.0$ \\
\hline \multicolumn{5}{|l|}{ W2 } \\
\hline IOP & $8.4 \pm 3.4$ & $10.2 \pm 2.9$ & $9.2 \pm 5.1$ & $9.4 \pm 4.4$ \\
\hline Medication & $0.0 \pm 0.0$ & $0.0 \pm 0.0$ & $0.0 \pm 0.0$ & $0.03 \pm 0.2$ \\
\hline \multicolumn{5}{|l|}{ W4 } \\
\hline IOP & $8.6 \pm 3.5$ & $12.7 \pm 6.2$ & $8.6 \pm 2.6$ & $13.7 \pm 5.4$ \\
\hline Medication & $0.0 \pm 0.0$ & $0.0 \pm 0.0$ & $0.12 \pm 0.5$ & $0.03 \pm 0.2$ \\
\hline \multicolumn{5}{|l|}{ M3 } \\
\hline IOP & $8.8 \pm 2.0$ & $11.0 \pm 2.9$ & $8.6 \pm 2.6$ & $12.9 \pm 5.4$ \\
\hline Medication & $0.03 \pm 0.2$ & $0.15 \pm 0.5$ & $0.12 \pm 0.5$ & $0.17 \pm 0.6$ \\
\hline \multicolumn{5}{|l|}{ M6 } \\
\hline IOP & $8.4 \pm 2.7$ & $12.2 \pm 3.5$ & $9.9 \pm 3.1$ & $13.0 \pm 3.7$ \\
\hline Medication & $0.06 \pm 0.2$ & $0.20 \pm 0.8$ & $0.21 \pm 0.6$ & $0.24 \pm 0.7$ \\
\hline \multicolumn{5}{|l|}{ M12 } \\
\hline IOP & $9.6 \pm 2.6$ & $13.8 \pm 3.8$ & $10.1 \pm 3.4$ & $12.8 \pm 3.7$ \\
\hline Medication & $0.29 \pm 0.8$ & $0.40 \pm 0.9$ & $0.21 \pm 0.6$ & $0.26 \pm 0.7$ \\
\hline
\end{tabular}

\section{Surgical complications and postoperative interventions}

No differences in rates of intra- or postoperative complications were detected (table 4). The use of MMC was not associated with a significant increase in complications in either the bevacizumab or the placebo group ( $\mathrm{p}$ range between 0.07 and 1). However, MMC was associated with a greater need for laser suture lysis in the bevacizumab group $(\mathrm{p}<0.05)$. A higher rate of needlings was performed in the placebo group (bevacizumab $8(12 \%)$ vs placebo $23(33 \%) ; \mathrm{p}=0.003))$. These were primarily performed in the first 3 months after surgery (bevacizumab 55 \pm 36 , range $28-100$ days; placebo $35 \pm 19$, range $8-70$ days; $\mathrm{p}=0.22$ ). No other difference in medical or surgical treatment implementation was recorded (table 5). Decision to re-start medication was made at similar average IOP levels in both groups (bevacizumab 18.5 $\pm 7.1 \mathrm{~mm} \mathrm{Hg}$, placebo $17.6 \pm 4.6 \mathrm{~mm} \mathrm{Hg}$; $\mathrm{p}=0.62$ ).

\section{DISCUSSION}

Our study describes a 12-month follow-up of 138 patients who underwent a primary trabeculectomy with or without an adjuvant bevacizumab injection peroperatively in the largest prospective randomised clinical trial in the field of anti-VEGF use for glaucoma surgery.

Patients who received a single anti-VEGF injection had a significantly higher rate of absolute success than those who received placebo, with a smaller number of bevacizumab-treated patients requiring further interventions (ie, needling) to achieve the required IOP reduction. Of note, the qualified success rates at 12 months follow-up were similar between the two treatment groups. Indeed, when the target IOP is not reached after trabeculectomy, rescue surgical interventions or re-initiation of antiglaucoma medication will be considered to reach this goal. As a result, the IOP at the final study visit (12 months) was similar, but at the cost of more frequent needlings in the placebo group. These results are in line with the existing clinical evidence suggesting that the use of anti-VEGF agents does not significantly enhance IOP reduction after trabeculectomy. ${ }^{12} 1617$

In this trial, intracameral injection was chosen over other alternative administration routes, such as intravitreal or subconjunctival injection. The latter have the disadvantage of further conjunctival manipulation, which may stimulate inflammation and fibroblast activation. Intracameral injections allow a diffuse distribution of the drug in the anterior chamber and throughout the outflow route into the filtration bleb without disturbing conjunctival integrity. Moreover, via this route of administration, bevacizumab may block the elevated aqueous VEGF concentrations that glaucoma patients are known to have, and which may contribute to the postoperative scarring process. ${ }^{71}$ Intravitreal injections might seem attractive for the possible 'depot effect' of the vitreous. However, in an animal study comparing the halflife of bevacizumab after subconjunctival versus intravitreal injection, the former had a considerably longer duration of action, possibly as a result of scleral binding of the anti-VEGF antibody. ${ }^{18}$ Finally, an intracameral injection using an already available paracentesis can avoid retinal complications associated with intravitreal administration, such as retinal detachment. ${ }^{19}$

Of note, in our study a single injection of bevacizumab was sufficient to significantly improve the outcome after trabeculectomy. It is known that the healing process after trabeculectomy takes several months, but that the maximal activity of the fibroblasts takes place in the first week postoperatively, ${ }^{20}$ and that the

Table 3 Comparison of success rates in both groups at the end of the 12-month period

\begin{tabular}{|c|c|c|c|c|}
\hline & Bevacizumab & Placebo & $p$ Value & OR (Cl) \\
\hline \multicolumn{5}{|c|}{ Trabeculectomy ( $n=138$ ) } \\
\hline Absolute success & $49 / 69(71 \%)$ & $35 / 69(51 \%)$ & 0.02 & 2.4 (1.1 to 5.1$)$ \\
\hline Qualified success & $60 / 69(87 \%)$ & $59 / 69(86 \%)$ & 0.99 & $1.1(0.4$ to 3.4$)$ \\
\hline \multicolumn{5}{|l|}{ Simple } \\
\hline Absolute success & $25 / 37(68 \%)$ & $17 / 35(49 \%)$ & 0.15 & 2.2 (0.8 to 6.4$)$ \\
\hline Qualified success & $31 / 37(84 \%)$ & $31 / 35(89 \%)$ & 0.74 & 0.67 (0.1 to 3.2$)$ \\
\hline \multicolumn{5}{|l|}{ Augmented } \\
\hline Absolute success & $24 / 32(75 \%)$ & $18 / 34(53 \%)$ & 0.08 & 2.7 (0.8 to 8.8$)$ \\
\hline Qualified success & $29 / 32(91 \%)$ & $28 / 34(82 \%)$ & 0.47 & $2.1(0.4$ to 13.9$)$ \\
\hline
\end{tabular}

Comparison between both treatment success rates based on Fisher's exact test using an intention to treat analysis. Augmented or simple trabeculectomy indicates whether an anti-metabolite (mitomycin C) was used, respectively. 
Figure 2 Kaplan-Meier survival curves showing the cumulative probability of success in both treatment groups during the 12-month period. Log-rank tests identified significant differences between the two groups when assessing absolute success $(p<0.01)$ but not in qualified success $(p=0.84)$.

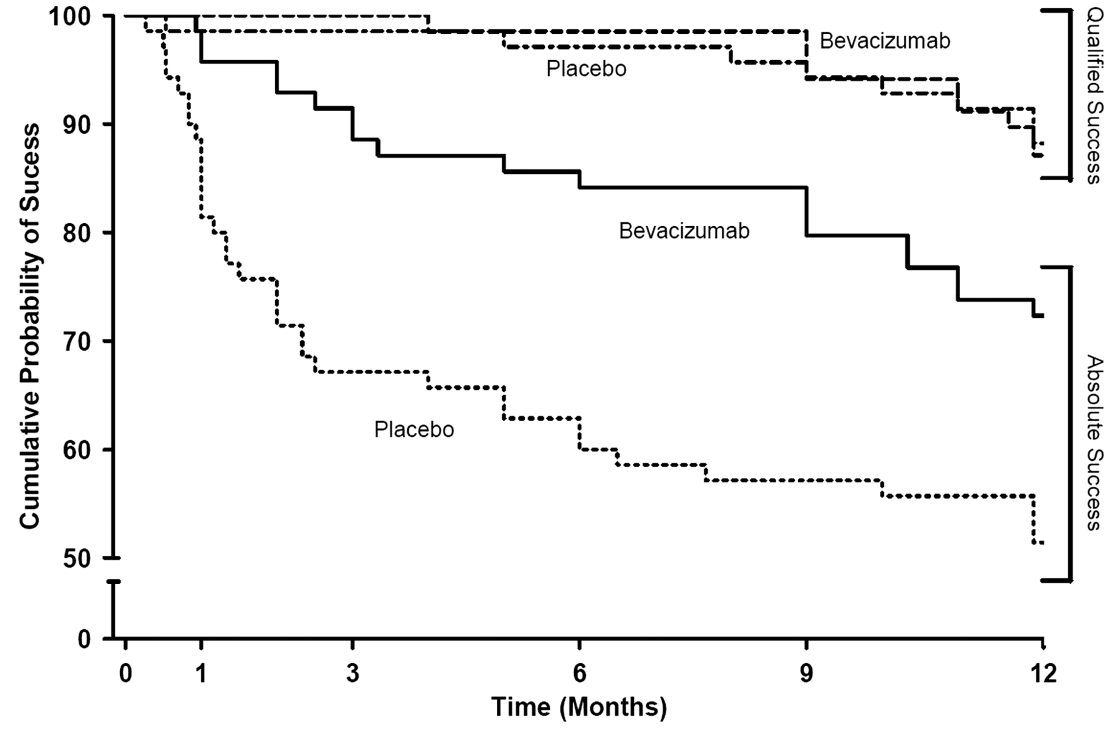

most typical time of cyst formation due to excessive scarring is 2-6 weeks postoperatively. ${ }^{21}$ While the half-life of bevacizumab in the human conjunctiva is not precisely known, animal studies have suggested that a single similar-dose conjunctival injection was sufficient to keep its levels above the minimal effective inhibitory concentration for over 8 weeks. ${ }^{18}$ As mentioned above, this may be related to scleral binding by these large antibody molecules, which would therefore allow a slow-release mechanism into the conjunctiva. ${ }^{22}$ This extended action of the anti-VEGF antibody might explain why a single injection was sufficient to have a significant effect on the postoperative healing process. Further studies are needed to investigate whether repeated administrations might further enhance this beneficial effect.

An important limitation of this study concerns the inclusion of patients scheduled for trabeculectomy with or without MMC use. Our initial study question was whether bevacizumab was a useful adjuvant to 'standard care' glaucoma surgery. When this study was initiated, the standard care at our centre for patients without increased risk of scarring involved the use of $\mathrm{MMC}$ only if a low target pressure was deemed necessary. This standard protocol was based on a Cochrane review on the subject, which suggests that MMC is particularly useful when aiming at very low IOPs. ${ }^{24}$ Therefore, it was considered unethical to withhold the use of anti-metabolites in those patients. However, to reduce the impact of this confounding factor, the randomisation process was designed as such that the number of patients receiving MMC was similar in the bevacizumab- and placebo-treated groups. Furthermore, although this study was not powered for analyses in subgroups, a post-hoc analysis revealed that despite no significant differences in the primary outcome at the 12-month period, bevacizumab could still significantly improve the survival curve in both subgroups (with or without MMC use). Importantly, this study was not designed to investigate whether bevacizumab could replace MMC or rather be used as an adjunctive to MMC, and therefore future studies will be

Table 4 Intra- and postoperative complications during follow-up

\begin{tabular}{|c|c|c|c|c|c|}
\hline & \multicolumn{2}{|c|}{ Bevacizumab $(n=69)$} & \multicolumn{2}{|c|}{ Placebo $(n=69)$} & \multirow[b]{2}{*}{ p Value } \\
\hline & MMC (n=32) & No MMC $(n=37)$ & MMC $(n=34)$ & No MMC $(n=35)$ & \\
\hline \multicolumn{6}{|l|}{ Intraoperative } \\
\hline Anaesthetic/systemic complications & $0(0 \%)$ & $0(0 \%)$ & $0(0 \%)$ & $1(3 \%)$ & 1.0 \\
\hline Subconjunctival haemorrhage & $1(3 \%)$ & $3(8 \%)$ & $0(0 \%)$ & $0(0 \%)$ & 0.12 \\
\hline Scleral flap problems & $0(0 \%)$ & $0(0 \%)$ & $0(0 \%)$ & $1(3 \%)$ & 1.0 \\
\hline Vitreous prolapse & $0(0 \%)$ & $0(0 \%)$ & $0(0 \%)$ & $1(3 \%)$ & 1.0 \\
\hline \multicolumn{6}{|l|}{ Postoperative } \\
\hline Choroidal detachment & $1(3 \%)$ & $4(11 \%)$ & $2(6 \%)$ & $3(9 \%)$ & 1.0 \\
\hline Hypotony >1 month & $7(22 \%)$ & $2(5 \%)$ & $6(18 \%)$ & $3(9 \%)$ & 1.0 \\
\hline Positive Siedel >1 month & $3(9 \%)$ & $5(14 \%)$ & $3(9 \%)$ & $3(9 \%)$ & 0.78 \\
\hline Hyphema & $0(0 \%)$ & $0(0 \%)$ & $2(6 \%)$ & $1(3 \%)$ & 0.24 \\
\hline Vitreous bleeding & $0(0 \%)$ & $0(0 \%)$ & $1(3 \%)$ & $0(0 \%)$ & 1.0 \\
\hline Corneal epitheliopathy or erosions & $0(0 \%)$ & $1(3 \%)$ & $3(9 \%)$ & $1(3 \%)$ & 0.37 \\
\hline Sterile vitritis & $1(3 \%)$ & $0(0 \%)$ & $0(0 \%)$ & $0(0 \%)$ & 1.0 \\
\hline Malignant glaucoma & $0(0 \%)$ & $1(3 \%)$ & $0(0 \%)$ & $0(0 \%)$ & 1.0 \\
\hline Cataract requiring surgery & $3(9 \%)$ & $2(5 \%)$ & $0(0 \%)$ & $2(6 \%)$ & 0.44 \\
\hline
\end{tabular}


Table 5 Postoperative medical and surgical interventions

\begin{tabular}{|c|c|c|c|c|c|}
\hline & \multicolumn{2}{|c|}{ Bevacizumab $(n=69)$} & \multicolumn{2}{|c|}{ Placebo $(n=69)$} & \multirow[b]{2}{*}{ p Value } \\
\hline & MMC $(n=32)$ & No MMC $(n=37)$ & MMC $(n=34)$ & No MMC $(n=35)$ & \\
\hline \multicolumn{6}{|l|}{ Surgical treatment } \\
\hline Suture lysis & 19 (59\%) & $10(27 \%)$ & $17(50 \%)$ & $12(34 \%)$ & 1.0 \\
\hline Needlings & $2(6 \%)$ & $6(16 \%)$ & $12(35 \%)$ & $11(31 \%)$ & 0.003 \\
\hline Bleb revision due to persistent Siedel & $0(0 \%)$ & $2(5 \%)$ & $1(3 \%)$ & $1(3 \%)$ & 1.0 \\
\hline Vitrectomy due to malignant glaucoma & $0(0 \%)$ & $1(3 \%)$ & $0(0 \%)$ & $0(0 \%)$ & 1.0 \\
\hline Intracameral tPA injection & $0(0 \%)$ & $0(0 \%)$ & $0(0 \%)$ & $1(3 \%)$ & 1.0 \\
\hline \multicolumn{6}{|l|}{ Medical treatment } \\
\hline Patients re-started on IOP-lowering drugs & $5(16 \%)$ & $6(16 \%)$ & $7(21 \%)$ & $8(23 \%)$ & 0.51 \\
\hline
\end{tabular}

needed to specifically answer those questions. Finally, while our study demonstrated that a $25 \mathrm{mg} / \mathrm{mL}$ bevacizumab injection administered peroperatively can induce a clinically relevant improvement in surgical outcomes, these beneficial effects of anti-VEGF could potentially be further optimised by using different dosing or treatment strategies (for example, more than one application of an anti-VEGF agent or slow-release preparations). More studies in this area are thus needed.

In conclusion, in this prospective randomised trial, bevacizumab used as an adjunctive to standard care trabeculectomy significantly increased the absolute success rate of trabeculectomy after 1 year of follow-up.

Contributors All the authors have been actively involved in the project, specifically: $\mathrm{EV}$, conduct/writing; LAP, data analysis/writing; TVB, reporting/data analysis; LS, data collection; SF, statistical analysis; LM, reporting/data discussion; WS, planning; $T Z$, planning, conduct; IS, planning, conduct and reporting. Furthermore, the authors EV, LAP and IS act as guarantors of the paper.

Funding This study was supported by a grant from the FRO "Funds for Research in Ophthalmology". EV was supported by a grant from the FWO "Fonds

Wetenschappelijk Onderzoek-Vlaanderen" and received a Pfizer Research Award in 2009 for this project.

Patient consent Obtained.

Ethics approval Institutional Review Board of the University Hospitals Leuven.

Provenance and peer review Not commissioned; externally peer reviewed.

\section{REFERENCES}

1 Seibold LK, Sherwood MB, Kahook MY. Wound modulation after filtration surgery. Surv Ophthalmol 2012;57:530-50.

2 Cordeiro MF, Schultz GS, Ali RR, et al. Molecular therapy in ocular wound healing. Br J Ophthalmol 1999;83:1219-24.

3 Rai $\mathrm{P}$, Kotecha $\mathrm{A}$, Kaltsos $\mathrm{K}$, et al. Changing trends in the incidence of bleb-related infection in trabeculectomy. Br J Ophthalmol 2012;96:971-5.

4 Mochizuki $\mathrm{K}$, Jikihara $\mathrm{S}$, Ando $\mathrm{Y}$, et al. Incidence of delayed onset infection after trabeculectomy with adjunctive mitomycin $\mathrm{C}$ or 5-fluorouracil treatment. $\mathrm{Br}$ J Ophthalmol 1997:81:877-83.

5 Kowanetz M, Ferrara N. Vascular endothelial growth factor signaling pathways: therapeutic perspective. Clin Cancer Res 2006;12:5018-22.

6 Wilgus TA, Ferreira AM, Oberyszyn TM, et al. Regulation of scar formation by vascular endothelial growth factor. Lab Invest 2008;88:579-90.
7 Li Z, Van Bergen T, Van de Veire S, et al. Inhibition of vascular endothelial growth factor reduces scar formation after glaucoma filtration surgery. Invest Ophthalmol Vis Sci 2009;50:5217-25.

8 O'Neill EC, Qin Q, Van Bergen NJ, et al. Antifibrotic activity of bevacizumab on human Tenon's fibroblasts in vitro. Invest Ophthalmol Vis Sci 2010;51:6524-32.

9 Tripathi RC, Li J, Tripathi BJ, et al. Increased level of vascular endothelial growth factor in aqueous humor of patients with neovascular glaucoma. Ophthalmology 1998; 105:232-7.

10 Memarzadeh F, Varma R, Lin LT, et al. Postoperative use of bevacizumab as an antifibrotic agent in glaucoma filtration surgery in the rabbit. Invest Ophthalmol Vis Sci 2009;50:3233-7.

11 Van Bergen T, Vandewalle $E$, Van de Veire $S$, et al. The role of different VEGF isoforms in scar formation after glaucoma filtration surgery. Exp Eye Res 2011;93:689-99.

12 Grewal DS, Jain R, Kumar $\mathrm{H}$, et al. Evaluation of subconjunctival bevacizumab as an adjunct to trabeculectomy: a pilot study. Ophthalmology 2008;115:2141-45.e2.

13 Gordon MO, Beiser JA, Brandt JD, et al. The Ocular Hypertension Treatment Study: baseline factors that predict the onset of primary open-angle glaucoma. Arch Ophthalmol 2002;120:714-20.

14 Vandewalle E, Van de Veire S, Renier $C$, et al. Trabeculectomy with or without anterior chamber maintainer and adjustable sutures. J Glaucoma Published Online First: 21 Aug 2012. doi:10.1097/JJG.0b013e318269806a

15 Breusegem C, Spielberg L, Van Ginderdeuren R, et al. Preoperative nonsteroidal anti-inflammatory drug or steroid and outcomes after trabeculectomy: a randomized controlled trial. Ophthalmology 2010;117:1324-30.

16 Jurkowska-Dudzińska J, Kosior-Jarecka E, Zarnowski T. Comparison of the use of 5-fluorouracil and bevacizumab in primary trabeculectomy: results at 1 year. Clin Experiment Ophthalmol 2012;40:e135-42.

17 Sedghipour MR, Mostafaei A, Taghavi Y. Low-dose subconjunctival bevacizumab to augment trabeculectomy for glaucoma. Clin Ophthalmol 2011;5:797-800.

18 Nomoto $\mathrm{H}$, Shiraga $\mathrm{F}$, Kuno N, et al. Pharmacokinetics of bevacizumab after topical, subconjunctival, and intravitreal administration in rabbits. Invest Ophthalmol Vis Sci 2009;50:4807-13.

19 Sampat KM, Garg SJ. Complications of intravitreal injections. Curr Opin Ophthalmol 2010;21:178-83.

20 Watanabe J, Sawaguchi S, Fukuchi T, et al. Effects of mitomycin C on the expression of proliferating cell nuclear antigen after filtering surgery in rabbits. Graefes Arch Clin Exp Ophthalmol 1997;235:234-40.

21 Schwartz AL, Van Veldhuisen PC, Gaasterland DE, et al. The Advanced Glaucoma Intervention Study (AGIS): 5. Encapsulated bleb after initial trabeculectomy. Am J Ophthalmol 1999;127:8-19.

22 Ambati J, Canakis CS, Miller JW, et al. Diffusion of high molecular weight compounds through sclera. Invest Ophthalmol Vis Sci 2000;41:1181-5.

23 Ambati J, Gragoudas ES, Miller JW, et al. Transscleral delivery of bioactive protein to the choroid and retina. Invest Ophthalmol Vis Sci 2000;41:1186-91.

24 Wilkins $M$, Indar A, Wormald R. Intra-operative mitomycin C for glaucoma surgery. Cochrane Database Syst Rev 2005;4:CD002897. doi:10.1002/14651858. CD002897.pub2 


\section{Intracameral bevacizumab as an adjunct to trabeculectomy: a 1-year prospective, randomised study}

Evelien Vandewalle, Luís Abegão Pinto, Tine Van Bergen, et al.

Br J Ophthalmol 2014 98: 73-78 originally published online October 24, 2013

doi: 10.1136/bjophthalmol-2013-303966

Updated information and services can be found at:

http://bjo.bmj.com/content/98/1/73.full.html

\section{These include:}

References This article cites 22 articles, 10 of which can be accessed free at: http://bjo.bmj.com/content/98/1/73.full.html\#ref-list-1

Email alerting Receive free email alerts when new articles cite this article. Sign up in service the box at the top right corner of the online article.

Topic Articles on similar topics can be found in the following collections

Collections

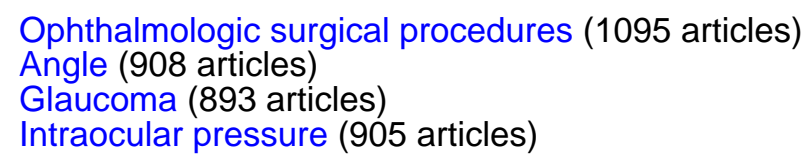

Notes

To request permissions go to:

http://group.bmj.com/group/rights-licensing/permissions

To order reprints go to:

http://journals.bmj.com/cgi/reprintform

To subscribe to BMJ go to:

http://group.bmj.com/subscribe/ 